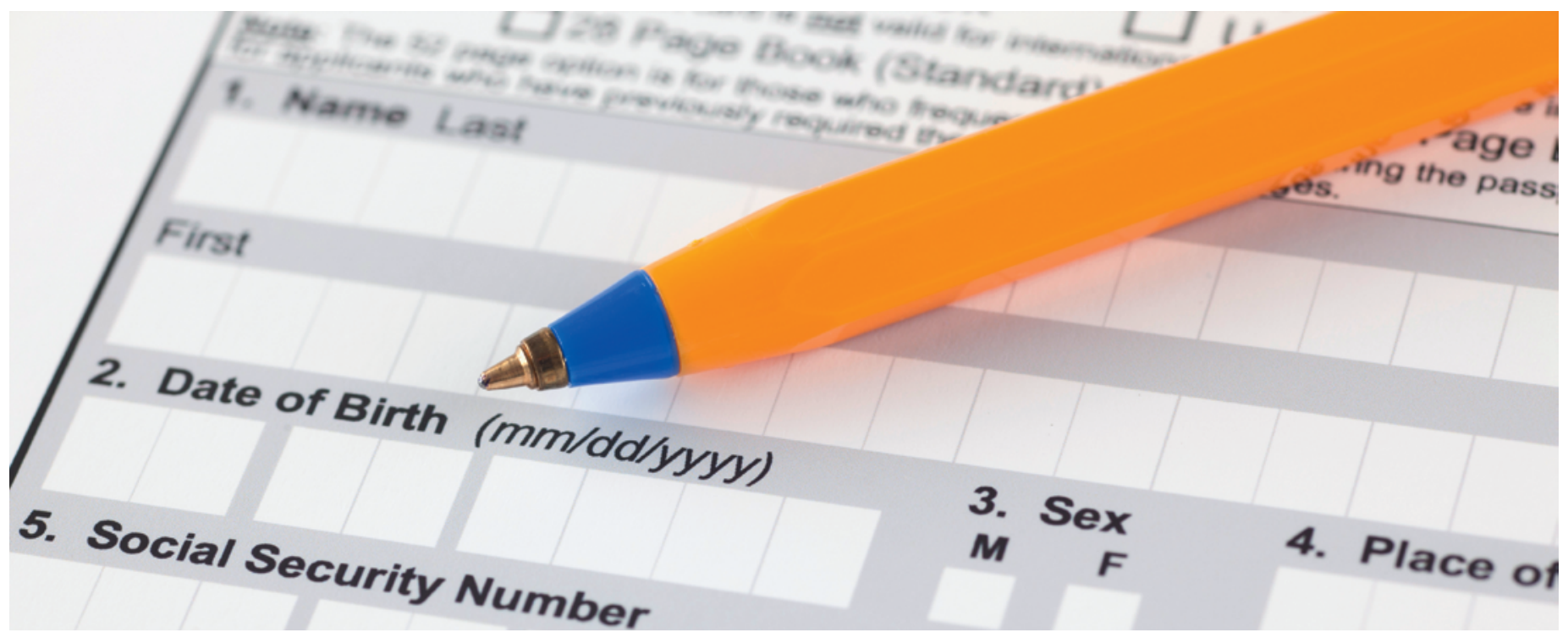

\title{
Altersbestimmung junger Migranten
}

\section{Sarah Depallens ${ }^{a}$, Fabienne Jäger ${ }^{b}$, Nicole Pellaud ${ }^{c}$}

a Dr. med., Service de pédiatrie CHUV, Lausanne, und Referenzgruppe Migration, Mitglied FMH

${ }^{b}$ Dr. med., Service de pédiatrie, Hôpital du Jura, Delémont, und Referenzgruppe Migranten, Mitglied FMH

c Dr. med., Service médicale scolaire et psychopédagogique, Sion, und Präsidentin SGP, Mitglied FMH

Diese Stellungnahme wurde durch Experten der betroffenen Fachrichtungen validiert:

Entwicklungspädiatrie: Prof. Oskar Jenni, Kinderspital Zürich; Pädiatrische Endokrinologie: Prof. Valérie Schwitzgebel, Universitätskinderklinik Genf;

Pädiatrische Radiologie: PD Dr. Georg Eich, Kantonsspital Aarau; SGP Referenzgruppe Migration und der Vorstand der SGP, www.swiss-paediatrics.org

1 Zwecks einfacherer Lesbarkeit wurde zusätzlich auf die weibliche Form verzichtet; sie ist aber selbstverständlich jeweils mitgemeint.

Die Schweizerische Gesellschaft für Pädiatrie (SGP) möchte die Ärzteschaft sowie die zuständigen eidgenössischen und kantonalen Behörden über die Problematik der Altersbestimmung bei jungen Migranten ${ }^{1}$ informieren. Sie publiziert eine Stellungnahme, die durch in Fragen der Gesundheit junger Migranten und Sozialpädiatrie spezialisierte Pädiater erarbeitet wurde. Die Stellungnahme soll die Betreuung junger Migranten, welche die Kinderrechte und die Menschenrechte ganz allgemein respektiert, in der Schweiz fördern.

\section{Mangels validierter Untersuchungsmetho- den können Ärzte sich nicht beteiligen}

Im Jahr 2016 empfing die Schweiz 27207 Asylbewerber, wovon 7,3\% als unbegleitete Minderjährige betrachtet wurden [1].

Nebst anderen Kriterien berücksichtigt das Asylverfahren das Alter des jungen Asylbewerbers; ab einem Alter von 18 Jahren besteht kein Anrecht mehr auf die den Minderjährigen von Amts wegen zuerkannte Betreuung (Wohn-/Kinderheim mit Erziehern, Schutz durch einen Vormund, Zugang zu einer Schule, ....). Dieser Unterschied hat einen wesentlichen
Einfluss auf die Zukunft des Jugendlichen. Der Zugang zu einer Ausbildung ist einer der wichtigsten Schutzfaktoren im Jugendalter. Ein Minderjähriger, der bei seiner Ankunft in der Schweiz als volljährig eingeschätzt wird, läuft somit von Anfang an Gefahr unge-

Um das Alter junger Menschen, die keine gültigen Ausweise haben, zu bestimmen, wenden sich die zuständigen Behörden in der Schweiz auch an die Ärzteschaft.

nügender sozialer Integration und damit einer gestörten Entwicklung. Um das Alter junger Menschen, die 
keine gültigen Ausweise haben, zu bestimmen, wenden sich die zuständigen Behörden in der Schweiz auch an die Ärzteschaft.

Aus medizinethischer, deontologischer Sicht darf eine Untersuchung nur durchgeführt werden, wenn 1) eine klare medizinische Indikation besteht, welche das Ziel einer Gesundheitsverbesserung unterstützt, 2) die Einverständniserklärung der aufgeklärten Person vorliegt, und es sich 3) um eine validierte Untersuchungsmethode ohne unnötiges Schädigungspotential handelt [2, 3].

Handelt es sich bei einer Untersuchung um einen nichttherapeutischen Auftrag (z.B. Asylbehörde), so sind umstrittene diagnostische Praktiken unzulässig und die betroffene Person ist klar zu informieren [3].

\section{Es gibt heutzutage keine wissenschaftliche Methode, die erlauben würde, das Alter eines 15- bis 20-Jährigen genau zu bestimmen.}

Wie dies jedoch mehrmals nachgewiesen und publiziert wurde $[4,5]$, sind die verwendeten Methoden, handle es sich nun um das Knochenalter [6-8, 11], körperliche Untersuchungen [8] oder die Beurteilung der Zahnentwicklung [9, 10, 12], zu approximativ, weisen weite Streubreiten auf und basieren auf oft nicht adaptierten Referenzwerten, welche weder die ethnische $[11,12]$ und sozioökonomische Vorgeschichte des Jugendlichen noch allfällige endokrinologische Pathologien genügend berücksichtigen, obwohl diese die Entwicklung des Jugendlichen beeinflussen können [8] Zudem sind die Verfahren mit unnötigen Strahlenbelastungen verbunden.

Es gibt heutzutage keine wissenschaftliche Methode, die erlauben würde, das Alter eines 15- bis 20-Jährigen genau $z u$ bestimmen und sicher zu entscheiden, ob er volloder minderjährig ist. So können Befunde, wie sie für Erwachsene typisch sind, durchaus auch in Minderjährigen gefunden werden, was zu einer Altersüberschätzung führen kann. Eine umfassende Abklärung des Jugendlichen durch Entwicklungsspezialisten bleibt sinnvoll, um dessen Entwicklungsstand und psychokognitiven Status zu bestimmen und ihn, wenn nötig, an eine sozialpädagogische Einrichtung weisen $\mathrm{zu}$ können, in der auf seine besondere Verwundbarkeit eingegangen werden kann [13].

Angesichts der Tatsache, das aktuell aus deontologischer Sicht die Grundlagen für besagte Altersbestimmungen nicht gegeben sind, diese jedoch wesentliche legale und soziale Konsequenzen für den Jugendlichen nach sich tragen kann, empfiehlt die Schweizerische Gesellschaft für Pädiatrie, wie dies schon mehrere internationale pädiatrische Gesellschaften und Akademien getan haben [14-16], ihren Mitgliedern und jedem anderen diesbezüglich angefragten Arzt, sich nicht an der Altersbestimmung junger Asylbewerber zu beteiligen. Wir schlagen vor, in diesem Sinne gegenüber den kantonalen Institutionen, die sich mit Migration befassen, Stellung zu nehmen.

\section{Literatur}

1 https://asile.ch/statistiques/suisse/

2 Charte du pédiatre, SSP 2017.

3 FMH-Standesordnung Art. 6, 8, http://www.fmh.ch/files/pdf18/ Standesordnung_August_2016_D.pdf

4 Aynsley-Green A, Cole TJ, Crawley H, Lessof N, Boag LR, Wallace RM. Medical, statistical, ethical and human rights considerations in the assessment of age in children and young people subject to immigration control. British medical bulletin. Jun 2012;102:17-42.

5 Hjern A, Brendler-Lindqvist M, Norredam M. Age assessment of young asylum seekers. Acta paediatrica (Oslo, Norway: 1992). Jan 2012;101(1):4-7.5.

6 Cole TJ. The evidential value of developmental age imaging for assessing age of majority. Annals of human biology. 2015;42(4):379-88.

7 Thodberg Hans Henrik, van Rijn Rick R, Jenni Oskar G, Martin David. D. Automated determination of bone age from hand Xrays at the end of puberty and its applicability for age estimation Int J Legal Med. DOI 10.1007/s00414-016-1471-8

8 Schwitzgebel V, Georg F. L'âge osseux ne permet pas de déterminer l'âge des jeunes requérants d'asile, Paediatrica Vol. 27 No. 3, 29, 2016.

9 Knell B. 2012. Zahnärztliche Altersdiagnostik zur Frage nach dem 18. Altersjahr. Kriminalistik-Schweiz. 2/12, 122-127, 2012

10 Reutimann, Felix. Zahnärztliche Altersdiagnostik: Untersuchung zur radiologischen Sichtbarkeit des Parodontalspaltes der ersten Molaren im Unterkiefer bei 14- bis 22-Jährigen. 2015, University of Zurich, Faculty of Medicine http://www.zora.uzh. ch/123990/1/Dissertation Reutimann 12 12 2015.pdf

11 Ontell FK, Ivanovic M, Ablin DS and Barlow TW. Bone age in children of diverse ethnicity, http://www.ajronline.org/doi/ abs/10.2214/ajr.167.6.8956565

12 Olze A, Schmeling A, Taniguchi M, Maeda H, van Niekerk P, Wernecke KD, Geserick G. Forensic age estimation in living subjects: the ethnic factor in wisdom tooth mineralization, Int J Legal Med. 2004 Jun;118(3):170-3. Epub 2004 Feb 6.

13 Messelken D, Crouse J. When childhood ends: estimating the age of young people. BMJ. 2015;351:h6699. doi: 10.1136/bmj.h6699

14 Royal College of Paediatrics and Child Health (2009) Policy: College statement on the role of paediatricians in the age assessment of unaccompanied young people seeking asylum, http:// www. rcpch.ac.uk/Policy

15 On behalf of the Advocacy and Ethics Group of the European Academy of Paediatrics, P.J.J. Sauer, A. Nicholson, D. Neubauer, Age determination in asylum seekers: physicians should not be implicated. Eur J Pediatr. Mar 2016; 175(3), 299-303 (2016).

16 International Society for Social Paediatrics Position Statement on Migrant Child Health 2017.

Bildnachweis

(c) Ekaterina79 | Dreamstime.com 